\title{
Agent-Based Evolutionary and Immunological Optimization
}

\author{
Aleksander Byrski and Marek Kisiel-Dorohinicki \\ Department of Computer Science, AGH University of Science and Technology, \\ Mickiewicz Avn. 30, 30-059 Cracow, Poland \\ \{olekb, doroh\}@agh. edu.pl
}

\begin{abstract}
An immunological selection mechanism for evolutionary multi-agent systems is discussed in the paper. It allows for reducing the number of fitness assignments required to get the solution of comparable quality as the classical resource-based selection in EMAS. Experimental studies aim at comparing the performance of immune-inspired selection, with resource-based one, and also with classical parallel evolutionary algorithms, based on typical multi-modal optimization benchmarks.
\end{abstract}

Keywords: multi-agent systems, evolutionary computation, artificial immune systems.

\section{Introduction}

The term evolutionary multi-agent systems (EMAS) covers a range of optimization techniques, which consist in the incorporation of evolutionary processes into a multiagent system at a population level. The most distinctive for these techniques are selection mechanisms, based on the existence of non-renewable resources, which is gained and lost when agents perform actions [6].

Even though the idea of EMAS proved working in a number of tests, it still reveals new features, especially when supported by specific mechanisms borrowed from other optimisation methods [7]. In particular, immunological approach was proposed as a more effective alternative to the classical resource-based selection used in EMAS [2]. The introduction of immune-based mechanisms may affect various aspects of the system behaviour, such as the diversity of the population and the dynamics of the whole process. From the practical point of view the most interesting effect is the substantial reduction of the number of fitness assignments required to get the solution of comparable quality. This is of vast importance for problems with high computational cost of fitness evaluation, like reverse problems involving simulation experiments to assess the solution, or hybrid soft computing systems with learning procedure associated with each assessment (e.g. evolution of neural network architecture for a particular problem) [1].

This paper focuses on the impact of the immune-based approach on the performance of EMAS applied to function optimization in comparison to the resource-based selection used alone. The results obtained for both agent-based approaches are also compared to classical parallel evolutionary algorithm (evolutionary algorithm with allopatric speciation).

Y. Shi et al. (Eds.): ICCS 2007, Part II, LNCS 4488, pp. 928 935, 2007.

(C) Springer-Verlag Berlin Heidelberg 2007 


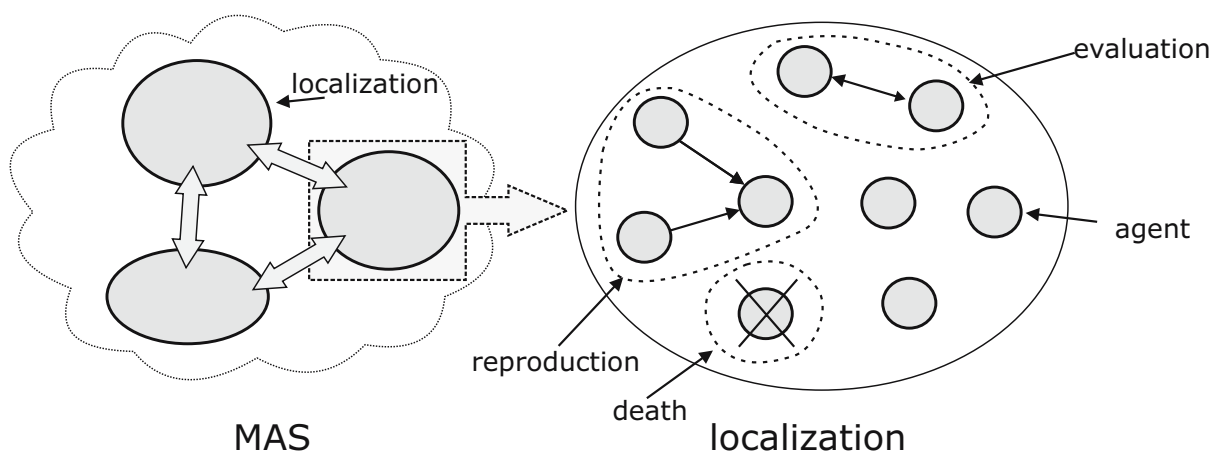

Fig. 1. Structure of the population and energetic selection principle in EMAS

Below, after a short presentation of the basics of evolutionary multi-agent systems and artificial immune systems, the details of the proposed approach are given. Then the selected results obtained for the optimization of typical multi-modal, multi-dimensional benchmark functions are presented.

\section{Evolutionary Multi-agent Systems}

In evolutionary multi-agent systems besides interaction mechanisms typical for agentbased systems (such as communication) agents are able to reproduce (generate new agents) and may die (be eliminated from the system). So inheritance may be accomplished by an appropriate definition of reproduction (with mutation and recombination), which is similar to classical evolutionary algorithms.

Unfortunately selection mechanisms known from classical evolutionary computation cannot be used in EMAS because of the assumed lack of global knowledge (which makes it impossible to evaluate all individuals at the same time), and the autonomy of agents (which causes that reproduction is achieved asynchronously). The resourcebased (energetic) selection scheme assumes that agents are rewarded for "good" behaviour, and penalized for "bad" behaviour (which behaviour is considered "good" or "bad" depends on the particular problem to be solved) [6].

In the simplest case the evaluation of an agent (its phenotype) is based on the idea of agent rendezvous. Assuming some neighbourhood structure (the simplest case would be population decomposition along with allopatric speciation model, see fig. 1) in the environment, agents evaluate their neighbours, and exchange energy. Worse agents (considering their fitness) are forced to give a fixed amount of their energy to their better neighbours. This flow of energy causes that in successive generations, survived agents should represent better approximations of the solution [4].

\section{Artificial Immune Systems}

Artificial immune systems, inspired by the human immunity, recently began to be the subject of increased researchers' interest. Different immune-inspired approaches were applied to many problems, such as classification or optimization [9]. 
The most often used algorithms, which are inspired by the immune system of vertebrates, are based on clonal and negative selection processes [5].

The algorithm of negative selection corresponds to its biological origin and consists of the following steps:

1. Lymphocytes are created, as yet they are considered immature.

2. The binding of these cells (affinity) to present self-cells (eg. good solutions of some problem) is evaluated.

3. Lymphocytes that bind themselves to "good" cells are eliminated.

4. Lymphocytes that survive are considered mature.

Mature lymphocytes are presented with the cells that have unknown origin (they may be self, or non-self cells), and they are believed to have possibility of classifying them [8]. The algorithm is usually used for classification problems.

The algorithm of clonal selection is based on biological process. Original approach used to classification problems was modified by De Castro and von Zuben [3] in order to adapt to solving optimization problems. In this approach the lymphocyte-antigen binding is represented by the value of fitness function (in fact, antigens may be identified with the function optima):

1. The population of lymphocytes is randomly created.

2. Every lymphocyte produces certain number of mutated clones.

3. Every lymphocyte in the population is replaced by the best of its clones (in the means of fitness function value) or retained.

4. The pairs of similar lymphocytes are selected (euclidean distance may be used for similarity measure) and from every pair better lymphocyte (in the means of fitness function value) is retained. Worse one is replaced by randomly generated new lymphocyte.

5. Steps 2-4 are repeated, until the stop condition is reached.

\section{Immunological Selection in EMAS}

In order to speed up the process of selection in EMAS, based on the assumption that "bad" phenotypes come from the "bad" genotypes, a new group of agents (acting as lymphocyte T-cells) may be introduced [21]. They are responsible for recognizing and removing agents with genotypes similar to the genotype pattern posessed by these lymphocytes. Other approach may introduce specific penalty applied by T-cells for recognized agents (certain amount of agent's energy is removed) instead of removing them from the system.

Of course there must exist some predefined affinity (lymphocyte-agent matching) function, which may be based on the percentage difference between corresponding genes. The agents-lymphocytes are created in the system after the action of death. The late agent genotype is transformed into lymphocyte patterns by means of mutation operator, and the newly created lymphocyte (or group of lymphocytes) is introduced into the system.

In both cases, new lymphocytes must undergo the process of negative selection. In a specific period of time, the affinity of immature lymphocytes patterns to "good" agents 


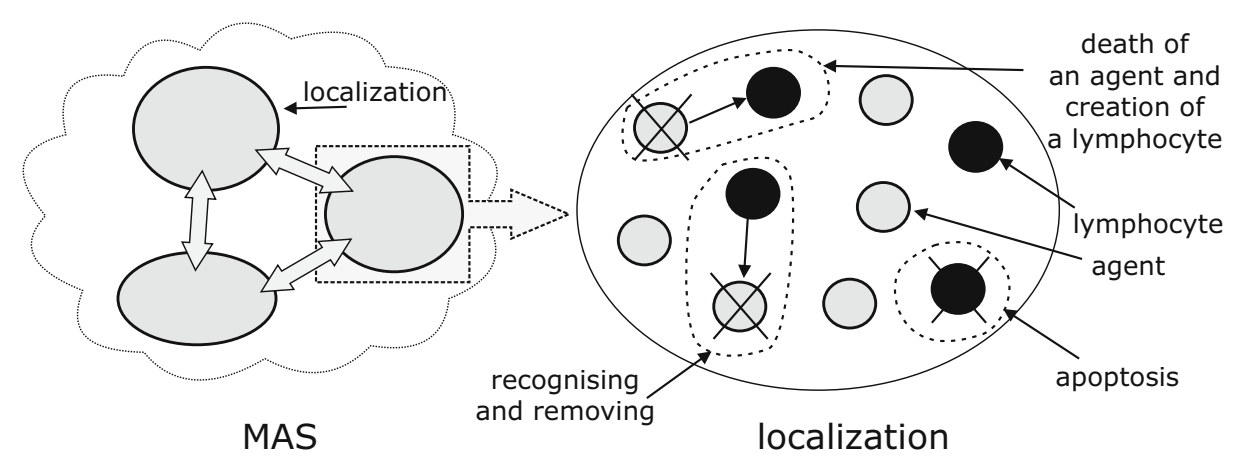

Fig. 2. Structure of the population and immunological selection principle in $i E M A S$

(posessing relative high amount of energy) is tested. If it is high (lymphocytes recognize "good" agents as "non-self") they are removed from the system. If the affinity is low, it is assumed that they will be able to recognize "non-self" individuals ("bad" agents) leaving agents with high energy intact.

The system working according to the above described principles will be called an immunological evolutionary multi-agent system (iEMAS) (see fig. 2).

\section{Configuration of the Examined Systems}

The systems were designed and implemented using distributed computational agent platform AgE developed at AGH University of Science and Technology 1 .

Much effort was put into configuring the examined systems in such way, that the obtained results may be comparable. It was of course a difficult task, because the agentbased and classical approaches were of different kind. For example, the mechanisms of selection are completely different, because there is no global knowledge in agent systems (therefore methods of selection are different).

Every algorithm was applied to optimization of 10-dimensional, well known benchmark functions, i.e. Rastrigin, Ackley, Griewank and De Jong. Real-value encoding was used. Every approach employed allopatric speciation, the populations were decomposed into 3 islands with initial population of 20 individuals. Discrete crossover operator along with uniform mutation with small macromutation probability $(0.1)$ were used. Every computation was performed during 10000 iterations.

Specific parameters for every examined algorithm will follow.

\section{Evolutionary algorithm}

- Tournament selection was used, because of its similarity to the rendezvous evaluation mechanism used in EMAS and iEMAS (the number of competing individuals was 7).

\footnotetext{
${ }^{1}$ http://age.iisg.agh.edu.pl
} 
- Crossover probability was 0.7 , mutation probability was 0.001 with 0.1 probability of macromutation.

- Every 20 iterations 5 the most different individuals were exchanged among the island in the process of migration.

\section{EMAS}

- Initial amount of agent's energy is 30. In every evaluation, agents exchanged 1 unit of energy.

- Agent was removed from the system, when its energy reached 0.

- Agent was able to reproduce, when its energy exceeded average energy computed for the evolutionary island.

- Agent was able to migrate when its energy exceeded about $110 \%$ of average energy computed for the evolutionary island.

\section{iEMAS}

- The pattern contained in T-cell has the same structure as the agent's genotype. The affinity function evaluates the difference between every element of genotype and pattern.

- The energy of T-cell was 150 . The T-cell was removed from the system after its energy reached 0 .

- The length of negative selection process was 30 iterations. The immature T-cell was removed from the system, after recognizing agent which energy exceeded the minimal energy needed for reproduction.

\section{Experimental Results}

The plots discussed below show averaged values (with the estimates of standard deviation) obtained for 20 runs of the systems with the same parameters.

In the figures 3(a) 3(b) 3(c) and 3(d) averaged best fitness is presented in consecutive steps of the system activity for EMAS, iEMAS and PEA for all optimization problems. These graphs show, that EMAS and iEMAS reached better or comparable results to PEA for three problems (Ackley, De Jong and Rastrigin). PEA turned out to be better only for Griewank problem. The final results of the optimization may be seen in the figure $4(\mathrm{a})$ and in the table 1

It seems that introduction of the immunological selection mechanism does not affect greatly the quality of obtained results. Both systems (EMAS and iEMAS) reached similar sub-optimal values in the observed period of time (continuation of the search would yield better results, however it was not the primary task of this research), although the results obtained for iEMAS were a little worse than its predecessor.

In the figure 4(b) the aggregated count of fitness function calls for Ackley problem is presented (other problems yielded very similar results, see table 2). It may be clearly seen, that the agent-based approaches require far less computations of fitness functions than PEA, what makes them good for solving problems where time of fitness computation is significant (e.g. reverse problems). Fitness count result for iEMAS was about $50 \%$ better than EMAS (see table 2). 


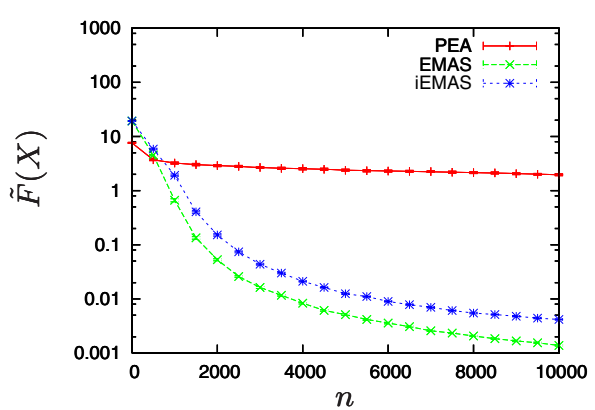

(a) Ackley problem optimization

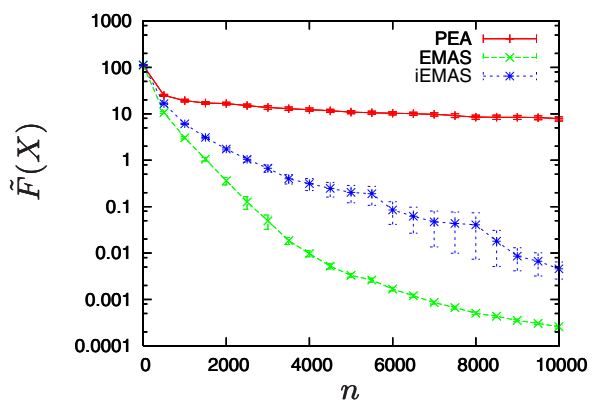

(c) Rastrigin problem optimization

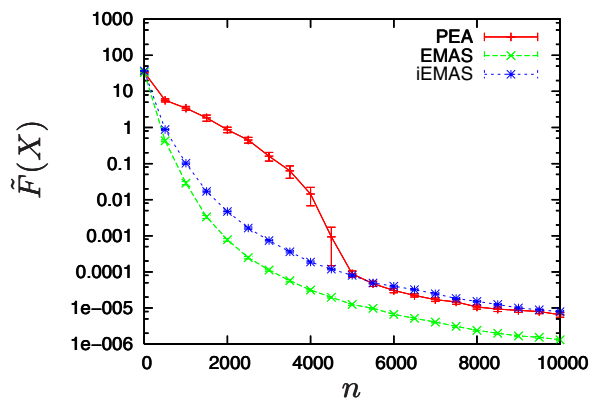

(b) De Jong problem optimization

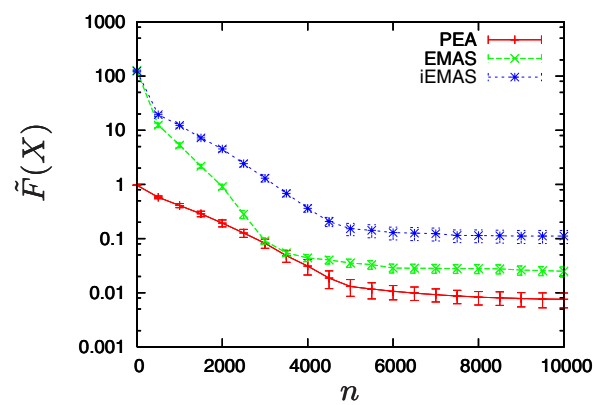

(d) Griewank problem optimization

Fig. 3. The best fitness in consecutive iterations

Table 1. The best fitness for EMAS, iEMAS and PEA in 10000 iteration

\begin{tabular}{|l|ll|l|ll|}
\hline Problem & PEA & & EMAS & \multicolumn{2}{l|}{ lEMAS } \\
\hline Ackley & 1.97 & \pm 0.07 & $1.38 \cdot 10^{-3} \pm 37 \cdot 10^{-6}$ & $4.17 \cdot 10^{-3}$ & $\pm 0.14 \cdot 10^{-3}$ \\
De Jong & $6.49 \cdot 10^{-6} \pm 0.96 \cdot 10^{-6}$ & $1.31 \cdot 10^{-6}$ & $\pm 68.6 \cdot 10^{-9}$ & $7.9 \cdot 10^{-6}$ & $\pm 0.59 \cdot 10^{-6}$ \\
Griewank & $7.58 \cdot 10^{-3} \pm 2.33 \cdot 10^{-3}$ & 0.02 & $\pm 5.05 \cdot 10^{-3}$ & 0.11 & \pm 0.03 \\
Rastrigin & 7.81 & \pm 0.81 & $0.26 \cdot 10^{-3} \pm 16.8 \cdot 10^{-6}$ & $4.58 \cdot 10^{-3} \pm 1.83 \cdot 10^{-3}$ \\
\hline
\end{tabular}

Table 2. The aggregated number of fitness function calls for EMAS, iEMAS and PEA in 10000 iteration

\begin{tabular}{|l|l|l|ll|}
\hline Problem & PEA & EMAS & \multicolumn{2}{|l|}{ iEMAS } \\
\hline Ackley & $0.6 \cdot 10^{6}$ & $57840 \pm 43$ & 25596 & \pm 149 \\
De Jong & $0.6 \cdot 10^{6}$ & $57769 \pm 43$ & 26172 & \pm 158 \\
Griewank & $0.6 \cdot 10^{6}$ & $58484 \pm 62$ & 30829 & \pm 379 \\
Rastrigin & $0.6 \cdot 10^{6}$ & $57787 \pm 29$ & 27727 & \pm 220 \\
\hline
\end{tabular}




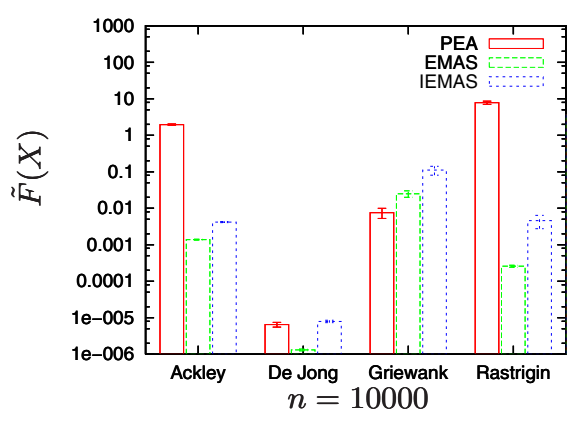

(a) The best fitness in 10000 iteration

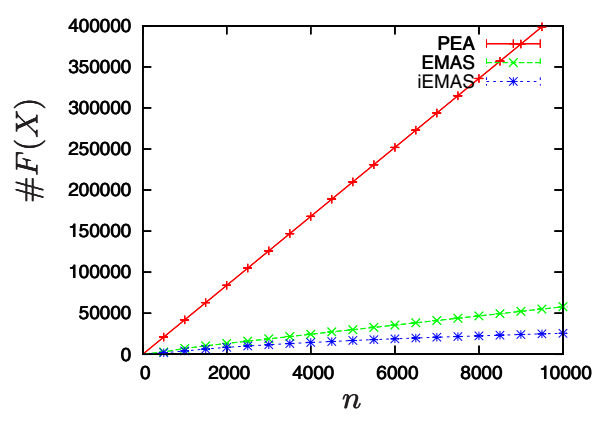

(b) Ackley problem fitness count

Fig. 4. The best fitness in 10000 iteration and aggregated number of fitness function calls

Relatively small value of standard deviation displayed in the presented graphs shows that the experimental results are trustworthy for examined problems.

\section{Conclusion}

In the paper immune-based selection mechanisms for evolutionary multi-agent systems were evaluated to show their performance in comparison to energetic ones and classical parallel evolutionary algorithms. As the experimental results show, it lowers the cost of the computation by decreasing the number of fitness assignments, though the results are comparable to these obtained for the system without immunological selection. Additionally it may be noticed that both agent-based approaches give better solutions than PEA in most considered problems.

\section{References}

1. A. Byrski and M. Kisiel-Dorohinicki. Immune-based optimization of predicting neural networks. In Vaidy S. Sunderam et. al., editor, Proc. of the Computational Science ICCS 2005 : 5th International Conference, Atlanta, GA, USA, Lecture Notes in Computer Science, LNCS 3516, pages 703-710. Springer Verlag, 2005.

2. A. Byrski and M. Kisiel-Dorohinicki. Immunological selection mechanism in agent-based evolutionary computation. In M. Kłopotek and et al., editors, Intelligent Information Processing and Web Mining : proceedings of the international IIS: IIPWM '05 conference : Gdańsk, Poland, June 1316, 2005, pages 411-415. Springer Verlag, 2005.

3. Leandro Nunes de Castro and Fernando J. Von Zuben. The clonal selection algorithm with engineering applications. In Artificial Immune Systems, pages 36-39, Las Vegas, Nevada, USA, 82000.

4. G. Dobrowolski and M. Kisiel-Dorohinicki. Management of evolutionary MAS for multiobjective optimization. Evolutionary Methods in Mechanics, pages 81-90, 2004.

5. W.H. Johnson, L.E. DeLanney, and T.A. Cole. Essentials of Biology. New York, Holt, Rinehart and Winston, 1969. 
6. M. Kisiel-Dorohinicki. Agent-oriented model of simulated evolution. In William I. Grosky and Frantisek Plasil, editors, SofSem 2002: Theory and Practice of Informatics, volume 2540 of LNCS. Springer-Verlag, 2002.

7. M. Kisiel-Dorohinicki, G. Dobrowolski, and E. Nawarecki. Agent populations as computational intelligence. In Leszek Rutkowski and Janusz Kacprzyk, editors, Neural Networks and Soft Computing, Advances in Soft Computing, pages 608-613. Physica-Verlag, 2003.

8. S. Wierzchoń. Artificial Immune Systems [in polish]. Akademicka oficyna wydawnicza EXIT, 2001.

9. S. Wierzchoń. Function optimization by the immune metaphor. Task Quaterly, 6(3):1-16, 2002 . 